Pacific Journal of Mathematics

INITIAL SEGMENTS OF DEGREES

May 1968 


\title{
INITIAL SEGMENTS OF DEGREES
}

\author{
Joseph G. ROSENSTEIN
}

Spector first constructed a function $h$ whose degree of recursive unsolvability is minimal-that is to say that any function recursive in $h$ is either recursive or of the same degree as $h$. Define a set $Q$ of degrees to be an initial segment of the upper semi-lattice of degrees of unsolvability if

$$
a \in Q \wedge b<a . \rightarrow . b \in Q .
$$

Spector's result can then be interpreted as saying that a certain partially ordered set occurs as an initial segment of the degrees; it was conjectured that the same is true for every finite partially ordered set which has a least member. Sacks then constructed two minimal degrees $a$ and $b$ such that $a \cup b$ has $a, b, 0$ as its only predecessors.

In this paper their methods are extended to obtain the following result. Let $T$ be the upper semi-lattice of all finite subsets of $N$. Then $T$ can be embedded as an initial segment of the degrees. From this it follows that any finite partial ordering which can be embedded as an initial segment of $P(B)$ (the power set of $B$ ), with $B$ finite, can also be embedded as an initial segment of the degrees.

We will define a function $h$ containing a countable infinity of functions $h_{i}$, each of minimal degree, such that $\underline{h}_{i_{1}} \cup \underline{h}_{i_{2}} \cup \cdots \cup \underline{h}_{i_{t}}$ will represent the finite subset $\left\{i_{1}, \cdots, i_{t}\right\}$ of $N$.

We first define a recursive function $\phi$ as follows: Let $\psi(k)=$ $(\mu n)((n+1)((n+1)+1) / 2)>k$ so that

$$
\frac{\psi(k)(\psi(k)+1)}{2} \leqq k<\frac{(\psi(k)+1)((\psi(k)+1)+1)}{2},
$$

and define $\phi(k)=k-(\psi(k)(\psi(k)+1)) / 2$. Then $\phi(k)$ takes on successively the values

$$
0,0,1,0,1,2,0,1,2,3, \cdots, n, 0,1,2, \cdots, n, n+1,0, \cdots \text {. }
$$

We will want to arrange things so that in the $k$ 'th interval of $g$, the $\phi(k)$ 'th function (carried on the powers of $p_{\phi(k)}$ ) will be the only one for which $f_{0}$ and $f_{1}$ have different values. (The reader who finds this sentence mysterious is encouraged to read the next few definitions and then return to this remark.)

Let $p_{i}$ be the $i$ 'th prime, and define recursive predicates $P_{i}$ and $P$ as follows:

$$
\begin{aligned}
P_{i}(x) & \equiv x=p_{i}^{(x) i} \\
P(x) & \equiv(E i) P_{i}(x) .
\end{aligned}
$$


Thus $P(x)$ if and only if $x$ is a prime-power.

A triple $\left(f_{0}, f_{1}, g\right)$ of functions is special if:

(i ) $(i)_{i<2}(t)\left[f_{i}(t)=0 \mathrm{~V} f_{i}(t)=1\right]$

(ii) $g(0)=0 \wedge(n)[g(n)<g(n+1)]$

(iii) $\sim P(x) \rightarrow f_{0}(x)=f_{1}(x)=0$

$$
\begin{aligned}
& (n)[(E x)\{g(n+1) \\
& \left.\quad \leqq x<g(n+2) \Lambda P_{\phi(n)}(x) \Lambda f_{0}(x) \neq f_{1}(x)\right\} \quad \Lambda \\
& \left.(z)\left\{g(n+1) \leqq z<g(n+2) \Lambda \sim P_{\phi(n)}(z) . \rightarrow . f_{0}(z)=f_{1}(z)\right\}\right] .
\end{aligned}
$$

If $\left(f_{0}, f_{1}, g\right)$ satisfies (i), (ii), and (iii) then we define $F\left(f_{0}, f_{1}, g\right)$ as follows:

$$
h \in F\left(f_{0}, f_{1}, g\right) \equiv(n)(E i)_{i<2}(x)\left[g(n) \leqq x<g(n+1) . \rightarrow . h(x)=f_{i}(x)\right] .
$$

If $\left(f_{0}, f_{1}, g\right)$ and $\left(u_{0}, u_{1}, v\right)$ are special triples, then we say that $\left(u_{0}, u_{1}, v\right)$ is a contraction of $\left(f_{0}, f_{1}, g\right)$ if:

(i ) $u_{0}, u_{1} \in F\left(f_{0}, f_{1}, g\right)$

(ii) $\quad(\mu t)\left(u_{0}(t) \neq u_{1}(t)\right)>(\mu t)\left(f_{0}(t) \neq f_{1}(t)\right)$

(iii) $(n)(E m)[v(n)=g(m)]$.

If $F\left(f_{0}, f_{1}, g\right)$ is defined, then we define $F^{*}\left(f_{0}, f_{1}, g\right)$ to be the set of all initial segments of members of $F\left(f_{0}, f_{1}, g\right)$-that is,

$$
\begin{aligned}
& s \in F^{*}\left(f_{0}, f_{1}, g\right) \equiv(E n)(g(n)=l h s) \\
& \quad \Lambda(m)_{g(m)<l h s}(E i)_{i<2}(x)\left[g(m) \leqq x<g(m+1) . \rightarrow .(s)_{x}=f_{i}(x)\right] .
\end{aligned}
$$

Lemma 1. Let $\left(f_{0}, f_{1}, g\right)$ be a special triple and let $s \in F^{*}\left(f_{0}, f_{1}, g\right)$. Then there is a special triple $\left(u_{0}, u_{1}, v\right)$ such that:

(i ) $\left(u_{0}, u_{1}, v\right)$ is a contraction of $\left(f_{0}, f_{1}, g\right)$

(ii) $u_{0}, u_{1}, v$ are recursive in $f_{0}, f_{1}, g$

(iii) $v(1)>l h s$

(iv) $(x)_{x<l h s}\left(u_{0}(x)=u_{1}(x)=(s)_{x}\right)$.

Proof. Let $m$ be such that $g(m)=l h s$. Define $v(0)=0, v(1)=$ $g(m+2), u_{0}(x)=u_{1}(x)=(s)_{x}$ for $x<l h s$, and $u_{0}(x)=u_{1}(x)=f_{0}(x)$ for $l h s \leqq x<g(m+2)$.

Suppose now that $v(n+1)$ is defined; we show how to define $v(n+2)$ and $u_{i}(x)$ for $v(n+1) \leqq x<v(n+2)$. Let $m^{*}$ be such that $g\left(m^{*}\right)=v(n+1)$ and let $r^{*}$ be such that

$$
r^{*}=(\mu r)\left(\phi(r)=\dot{\phi}(n) \wedge r+1 \geqq m^{*}\right) \text {. }
$$

Then define $v(n+2)=g\left(r^{*}+2\right)$. We also define $u_{i}(t)$ for $v(n+1) \leqq$ $t<v(n+2)$ as follows:

$$
\begin{array}{ll}
u_{i}(t)=f_{0}(t) & \text { for } v(n+1) \leqq t<g\left(r^{*}+1\right) \\
u_{i}(t)=f_{i}(t) & \text { for } g\left(r^{*}+1\right) \leqq t<v(n+2) .
\end{array}
$$


It is clear that the triple $\left(u_{0}, u_{1}, v\right)$ is special and has the properties (i)-(iv).

Let $0 \leqq a_{0}<a_{1}<a_{2}<\cdots<a_{k-1}$ be a finite sequence of integers and let $h$ be any function whose range is $\{0,1\}$. We define $h_{a_{0} \cdots a_{k-1}}$ as follows:

$$
\begin{aligned}
h_{a_{0} \cdots a_{k-1}}(x) & =0 \quad \text { if } \sim\left(P_{a_{0}}(x) \vee \cdots \vee P_{a_{k-1}}(x)\right) \\
& =h(x) \quad \text { if } P_{a_{0}}(x) \vee \cdots \vee P_{a_{k-1}}(x)
\end{aligned}
$$

Where there is no danger of ambiguity we will abbreviate $h_{a_{0} \cdots a_{k-1}}$ to $h_{-}$. We also use the convention that $x_{0}, \cdots, \hat{x}_{j}, \cdots, x_{r}$ means $x_{0}, \cdots, x_{j-1}, x_{j+1}, \cdots, x_{r}$.

We construct a function $h$ so that for each $j$, the function $h_{j}$ is of minimal degree. The degree $\underline{h}_{j}$ will correspond to the finite set $\{j\}$. We see that $\underline{h}_{a_{0}} \cup \cdots \cup \underline{h}_{a_{k-1}}=\underline{h}_{a_{0} \cdots a_{k-1}}$, so we will assign $\underline{h}_{a_{0} \cdots a_{k-1}}$ to be the degree corresponding to the set $\left\{a_{0}, \cdots, a_{k-1}\right\}$. Let $A$ be a finite subset of $N$, and let $A_{1}, \cdots, A_{t}$ be all sets obtained by deleting one element from $A$. The lemma below is concerned with guaranteeing the degree analog of the following statement: If $B \leqq A$, then either $B=A$ or for some $j, B \leqq A_{j}$.

Note that if $\left\{a_{0}, \cdots, a_{k-1}\right\}=\dot{\phi}$, then $h_{a_{0} \cdots a_{k-1}} \equiv 0$.

Lemma 2. Let $\left(f_{0}, f_{1}, g\right)$ be a special triple and let $e$ and $0 \leqq$ $a_{0}<a_{1}<\cdots<a_{k-1}$ be given. Then there is a special triple $\left(u_{0}, u_{1}, v\right)$ such that:

(i) $\left(u_{0}, u_{1}, v\right)$ is a contraction of $\left(f_{0}, f_{1}, g\right)$

(ii) $u_{0}, u_{1}, v$ are recursive in $f_{0}, f_{1}, g$

(iii) if $h \in F\left(u_{0}, u_{1}, v\right)$ then either

(A) $\{e\}^{h}-(n)$ is undefined for some $n$

or

$\left(B_{j}\right) \quad\{e\}^{h}-(\imath)$ is recursive in $h_{a_{0} \cdots \hat{a}_{j} \cdots a_{k-1}}, f_{0}, f_{1}, g$

$$
\text { for some } j, 0 \leqq j \leqq k-1
$$

or

(C) $h_{-}$is recursive in $\{e\}^{h}-, f_{0}, f_{1}, g$.

Proof. Consider $F\left(f_{0_{-}}, f_{1_{-}}, g\right)$, which is precisely

$$
\left\{h_{a_{0} \cdots a_{k-1}} \mid h \in F\left(f_{0}, f_{1}, g\right)\right\},
$$

and denote $F^{*}\left(f_{0_{-}}, f_{1_{-}}, g\right)$ by $F^{*}$. 
Case 1.

$(E s)(E n)(w)\left[s \in F^{*} \Lambda\left(w \in F^{*} \Lambda w \operatorname{ext} s . \rightarrow\left\{\{\}^{w}(n)\right.\right.\right.$ is undefined $\left.)\right]$.

Let $s_{0}, n_{0}$ be the least pair satisfying the case hypothesis. Let

$$
\begin{aligned}
s= & (\mu w)\left(w \in F^{*}\left(f_{0}, f_{1}, g\right) \Lambda l h w=l h s_{0}\right. \\
& \left.\Lambda(x)_{x<1 h s_{0}}\left(P_{a_{0}}(x) \bigvee \cdots \bigvee P_{a_{k-1}}(x) . \rightarrow(w)_{x}=\left(s_{0}\right)_{x}\right)\right) .
\end{aligned}
$$

Apply Lemma 1 to get a special triple $\left(u_{0}, u_{1}, v\right)$ satisfying (i) and (ii). We need only show that $\left(u_{0}, u_{1}, v\right)$ also satisfies (iii) $(A)$.

But if $h \in F\left(u_{0}, u_{1}, v\right)$ and $\{e\}^{h}-\left(n_{0}\right)$ is defined, then there is an initial segment $w$ of $h_{-}$such that $\{e\}^{w}\left(n_{0}\right)$ is defined. But $s$ is an initial segment of $h$; and if $w$ ext $s_{0}$ then $\{e\}^{w}\left(n_{0}\right)$ is undefined and if $s_{0}$ ext $w$ then $\{e\}^{s_{0}}\left(n_{0}\right)$ is defined. Both of these are impossible. Hence $\{e\}^{h}-\left(n_{0}\right)$ is undefined.

Case $2_{j}$. The hypothesis of Case 1 is false and in addition:

$$
\begin{aligned}
(E s)(n)(u)(v)\left[s \in F^{*}\right. & \Lambda\left\{u \in F^{*} \Lambda v \in F^{*} \Lambda l h u=l h v\right. \\
& \Lambda\{e\}^{n}(n) \text { is defined } \Lambda u \operatorname{ext} s \\
& \Lambda\{\}^{v}(n) \text { is defined } \Lambda v \operatorname{ext} s \\
\Lambda(x)_{l h s \leqq x<l h u}\left(P_{a_{0}}(x) \bigvee \cdots \bigvee \hat{P}_{a_{j}}(x) \bigvee \cdots\right. & \cdots P_{a_{k-1}}(x) \\
. \rightarrow & \left.\left.\left.(u)_{x}=(v)_{x}\right)\right\} \rightarrow\{e\}^{u}(n)=\{e\}^{v}(n)\right]
\end{aligned}
$$

This hypothesis will be referred to as statement $(j)$.

Let $s_{0}$ be the least $s$ satisfying the statement $(j)$, where $j$ is the smallest $i$ such that statement $(i)$ holds. Let

$$
\begin{aligned}
& s=(\mu w)\left(w \in F^{*}\left(f_{0}, f_{1}, g\right) \Lambda \operatorname{lh} w=l h s_{\sigma}\right. \\
& \Lambda(x)_{x<l h s_{0}}\left(P_{a_{0}}(x) \bigvee \cdots \vee P_{a_{k-1}}(x) . \rightarrow(w)_{x}=\left(s_{0}\right)_{x}\right) .
\end{aligned}
$$

Apply Lemma 1 to get a special triple $\left(u_{0}, u_{1}, v\right)$ satisfying (i) and (ii). We show that $\left(u_{0}, u_{1}, v\right)$ also satisfies (iii) $\left(B_{j}\right)$.

So let $h \in F\left(u_{0}, u_{1}, v\right)$ be such that $\{e\}^{h}-(n)$ is defined for all $n$. Then $s_{0}$ must be an initial segment of $h_{-}$. We show how to compute $\{e\}^{h-}(n)$ from $f_{0}, f_{1}, g, h_{a_{0} \cdots \hat{a}_{j} \cdots a_{k-1}}$.

Now since $\{e\}^{h}-(n)$ is defined, $h_{-} \in F\left(f_{0_{-}}, f_{1_{-}}, g\right)$, and the hypothesis of Case 1 is false, there is a $w \in F^{*}$ such that $w \operatorname{ext} s_{0} \Lambda\{e\}^{w}(n)$ is defined and

$$
(x)_{l h s \leqq x<l h w}\left(P_{a_{0}}(x) \mathrm{V} \cdots \mathrm{V} \hat{P}_{a_{j}}(x) \mathrm{V} \cdots \bigvee P_{a_{k-1}}(x) . \rightarrow_{.}(w)_{x}=h(x)\right) .
$$

One can easily find such a $w$ by examining sufficiently large segments of $f_{0}, f_{1}, g$, and $h_{a_{0} \cdots \hat{a}_{j} \cdots a_{k-1}}$. We claim that $\{e\}^{w}(n)=\{e\}^{h}-(n)$. Indeed, if $v$ is an initial segment of $h_{-}$such that $v \in F^{*} \Lambda v \operatorname{ext} s_{0} \Lambda\{e\}^{v}(n)$ 
is defined $\Lambda\{e\}^{v}(n)=\{e\}^{h}-(n)$, then $\{e\}^{v}(n)=\{e\}^{w}(n)$ by statement $(j)$. Hence $\{e\}^{w}(n)=\{e\}^{h}-(n)$ and so we have computed $\{e\}^{h-}(n)$ from $f_{0}, f_{1}, g, h_{a_{0} \cdots \hat{a}_{j} \cdots a_{k-1}}$; thus $\{e\}^{h-}$ is recursive in these functions.

Case 3. Case 1 and Case 2 are false for all $j$. We claim that for each $j, 0 \leqq j \leqq k-1$, following statement $\left(j^{\prime}\right)$ holds:

$(s)(y)(E n)(E u)(E w)\left[s \in F^{*} \wedge y \in F^{*} \Lambda l h s=l h y\right.$

$\rightarrow\left\{u \in F^{*} \Lambda w \in F^{*} \Lambda u \operatorname{ext} s \Lambda w \operatorname{ext} y\right.$

$\Lambda\{e\}^{u}(n)$ is defined $\Lambda\{e\}^{w}(n)$ is defined $\Lambda l h u=l h w$

$\Lambda\{e\}^{u}(n) \neq\{e\}^{w}(n) \Lambda(x)_{l h s \leq x<l h u}\left(P_{a_{0}}(x) \bigvee \cdots \vee\right.$

$\left.\left.\left.\hat{P}_{a_{j}}(x) \bigvee \cdots \bigvee P_{a_{k-1}}(x) . \rightarrow(u)_{x}=(w)_{x}\right)\right\}\right]$.

To prove $\left(j^{\prime}\right)$ suppose $s, y \in F^{*}$. Since statement $(j)$ is false there is an $n$, a $u^{\prime}$ and a $u^{\prime \prime}$ such that $u^{\prime}, u^{\prime \prime} \in F^{*}$ and $l h u^{\prime}=l h u^{\prime \prime}$ and $\{e\}^{w^{\prime}}(n)$ is defined and $\{e\}^{u^{\prime \prime}}(n)$ is defined and $u^{\prime}$ extends $s$ and $u^{\prime \prime}$ extends $s$ and $\{e\}^{u^{\prime}}(n) \neq\{e\}^{u^{\prime \prime}}(n)$ and

$$
(x)_{l h s \leqq x<l h u}\left(P_{a_{0}}(x) \bigvee \cdots \vee \hat{P}_{a_{j}}(x) \bigvee \cdots \vee P_{a_{k-1}}(x) . \rightarrow .\left(u^{\prime}\right)_{x}=\left(u^{\prime \prime}\right)_{x}\right) .
$$

Let $y^{\prime}$ be defined by $\left(y^{\prime}\right)_{x}=(y)_{x}$ for $x<$ lhy and $\left(y^{\prime}\right)_{x}=\left(u^{\prime}\right)_{x}$ for $l h y \leqq x<l h u^{\prime}$. Then since Case 1 is false there is a $w$ such that $w \in F^{*}$ and $w$ extends $y^{\prime}$ and $\{e\}^{w}(n)$ is defined. Now either $\{e\}^{u^{\prime}}(n)$ or $\{e\}^{u^{\prime \prime}}(n)$ is different from $\{e\}^{w}(n)$; suppose without loss of generality that the first one is. Define $u$ by $(u)_{x}=\left(u^{\prime}\right)_{x}$ for $x<l h u^{\prime}$ and $(u)_{x}=$ $(w)_{x}$ for $l h u^{\prime} \leqq x<l h w$. Then $n, u$, and $w$ have the properties described in statement $\left(j^{\prime}\right)$.

We will use the truth of these statements $\left(j^{\prime}\right)$ to define $u_{0}, u_{1}$, and $v$.

Let $m^{*}=(\mu n)\left(f_{0}(n) \neq f_{1}(n)\right)$. Let $v(0)=0, v(1)=g\left(m^{*}+1\right)$, and $u_{i}(m)=f_{0}(m)$ when $m<v(1)$. Fix $t \geqq 0$ and suppose that $v(m)$ has been defined for $m \leqq t+1$, that $v(0)<v(1)<\cdots<v(t+1)$, and that $u_{i}(m)$ has been defined for all $m<v(t+1)$. We shall define $v(t+2)$ and $u_{i}(m)$ for $v(t+1) \leqq m<v(t+2)$.

We must first examine $t$ to decide which of the infinite number of functions should be the one varying in this interval. If $\phi(t)=a_{j}$ for some $j$ we shall use statement $\left(j^{\prime}\right)$; if $\phi(t) \neq a_{j}$ for all $j$ we shall use statement $\left(0^{\prime}\right)$.

For each $i \leqq 2^{t+1}$ we define a pair $\left(x_{i}, y_{i}\right)$ of partial functions with finite domains. Let $x_{0}$ and $y_{0}$ be the partial functions whose domain is empty. Fix $i$ so that $0<i \leqq 2^{t+1}$ and suppose that $\left(x_{i-1}, y_{i-1}\right)$ has been defined. Let $i=c_{0} 2^{0}+c_{1} 2^{1}+\cdots+c_{t+1} 2^{t+1}$ where each $c_{j}$ is either 0 or 1 . We assume that domain of $x_{i-1}=$ domain of $y_{i-1}=$ $\{m \mid v(t+1) \leqq m<z\}$ where $z=g(r)$ for some $r$. 
We define two initial segments, $s$ and $y$ :

$$
\begin{array}{ll}
s(m)=y(m)=u_{a-}(m) & \text { if } v(j) \leqq m<v(j+1), c_{j}=a, \text { and } j \leqq t ; \\
s(m)=x_{i-1}(m) & \text { if } v(t+1) \leqq m<z ; \\
y(m)=y_{i-1}(m) & \text { if } v(t+1) \leqq m<z .
\end{array}
$$

Hence by the appropriate assumption $\left(j^{\prime}\right)$ there is a natural number $n$ and segments $u, w$ such that $s, y, n, u, w$ have the properties described in statement $\left(j^{\prime}\right)$. Choose these minimal. Define $x_{i}(m)=$ $u(m)$ and $y_{i}(m)=w(m)$ for all $m$ such that $v(t+1) \leqq m<l h u$. The assumptions we made concerning $x_{i-1}$ and $y_{i-1}$ remain true when $i-1$ is replaced by $i$. We thus proceed to get $x_{t^{*}}$ and $y_{t^{*}}$ where $t^{*}=2^{t+1}$.

Let $m_{0}$ be such that $g\left(m_{0}\right)=v(t+1)+$ (cardinality of domain of $\left.x_{t^{*}}\right)$, and let $r_{0}=(\mu r)\left(\phi(r)=\dot{\phi}(t) \Lambda g(r+1) \geqq g\left(m_{0}\right)\right)$ and define $v(t+2)=g\left(r_{0}+2\right)$.

If $\phi(t)=a_{j}$, then we want variation in the $a_{j}$ 'th function in this interval, so define

$$
\begin{aligned}
u_{0}(m)=\left\{( \mu s ) \left(s \in F^{*}\left(f_{0}, f_{1}, g\right) \Lambda l h s=g\left(m_{0}\right) \Lambda\right.\right. \\
\left.(x)_{v(t+1) \leqq x<l h s}\left(P_{a_{0}}(x) \bigvee \cdots \vee P_{a_{k-1}}(x) . \rightarrow .(s)_{x}=\left(x_{t^{*}}\right)_{x}\right)\right\}_{m},
\end{aligned}
$$

and $u_{1}(m)$ to be the same except that $y_{t^{*}}$ replaces $x_{t^{*}}$, for all $m$ such that $v(t+1) \leqq m<g\left(m_{0}\right)$. Furthermore, for $g\left(m_{0}\right) \leqq m<g\left(r_{0}+1\right)$, let $u_{0}(m)=u_{1}(m)=f_{0}(m) ;$ and for $g\left(r_{0}+1\right) \leqq m<g\left(r_{0}+2\right)$, let $u_{i}(m)=f_{i}(m)$.

If $\phi(t) \neq a_{j}$ for any $j$, then we want one of the other functions to vary on this interval, so we define $u_{0}$ and $u_{1}$ exactly as above except that for $v(t+1) \leqq m<g\left(m_{0}\right)$, both $u_{0}(m)$ and $u_{1}(m)$ are equal to the expression with $x_{t^{*}}$.

The assumptions we made concerning the values of $u_{i}(m)$ for $m<v(t+1)$ and $i<2$ remain true when $t+1$ is replaced by $t+2$. We thus proceed to get functions $u_{0}, u_{1}, v$ such that $\left(u_{0}, u_{1}, v\right)$ is clearly a special triple and satisfies $(i)$.

We need only show that $\left(u_{0}, u_{1}, v\right)$ satisfies (ii) and (iii) $(C)$. As for the first, we must show that at each stage of the construction of $u_{0}, u_{1}, v$ the choices made are made recursively in $f_{0}, f_{1}, g$ and that this is done uniformly (with respect to the stages.) The latter is clear since what is done between $v(n+1)$ and $v(n+2)$ depends only on $\dot{\phi}(n)$. As to the former, we choose, for given $s$ and $y$, an $n$, a $u$, and a $v$ according to statement $\left(j^{\prime}\right)$. We show that this is done recursively in $f_{0}, f_{1}, g$. Indeed $\left(j^{\prime}\right)$ can be written in the form $(s)(y)(E n)(E u)(E w)\left(E y^{\prime}\right)\left(E y^{\prime \prime}\right) Q\left(s, y, n, u, w, y^{\prime}, y^{\prime \prime}\right)$ where $y^{\prime}$ and $y^{\prime \prime}$ are to be considered as Godel numbers of computations for $\{e\}^{u}(n)$ and $\{e\}^{w}(n)$ and the predicate $Q$ is recursive in $f_{0}, f_{1}, g$. To make our 
choice we look successively at quintuples $\left(n, u, w, y^{\prime}, y^{\prime \prime}\right)$ until we find one that works. Thus with the stipulation that where we said above "choose $n, u, w$ minimal" we meant "choose $n, u, w$ so that $\left(n, u, w, y^{\prime}, y^{\prime \prime}\right)$ is minimal," it is clear that $u_{0}, u_{1}, v$ are recursive in $f_{0}, f_{1}, g$.

To show that (iii) $(C)$ holds, we suppose that $h \in F\left(u_{0}, u_{1}, v\right)$ and that $\{e\}^{h}-(m)$ is defined for all $m$. We must show that $h_{-}$is recursive in $f_{0}, f_{1}, g,\{e\}^{h}-$. Fix $t \geqq 0$. We indicate how to obtain the values of $h_{-}(m)$ for $v(t+1) \leqq m<v(t+2)$ from $f_{0}, f_{1}, g,\{e\}^{h_{-}}$, and the values of $h_{-}(m)$ for $m<v(t+1)$. Note that the values of $h_{-}(m)$ for $m<v(1)$ are obtained quite easily from $f_{0}, f_{1}$, and $g$.

Now since $h \in F\left(u_{0}, u_{1}, v\right)$ it follows that for each $j<t+1$ there is an $a$ such that $(m)\left(v(j) \leqq m<v(j+1) . \rightarrow_{.} h_{-}(m)=u_{a-}(m)\right)$. For each $j$, let $c_{j}$ be the least $a$ for which this holds. Let $i=2^{t+1}$ if $(j)\left(j<t+1 . \rightarrow c_{j}=0\right)$ and otherwise let $i=c_{0} 2^{0}+c_{1} 2^{1}+\cdots+c_{t} 2^{t}$.

Consider now the definition of $x_{i}$ and $y_{i}$ in Case 3 . Clearly $s(m)=$ $y(m)=h_{-}(m)$ for $m<v(t+1)$. Also $\{e\}^{u}(n)$ and $\{e\}^{w}(n)$ are defined and are unequal, and $u, w$, and $n$ can be computed from $f_{0}, f_{1}, g$ and the values of $h_{-}(m)$ for $m<v(t+1)$; this is so because $Q$ is recursive. Now either $u$ or $w$ is an initial segment of $h_{-}$, and knowing which one determines what $h_{-}(m)$ is on all of the interval $v(t+1) \leqq$ $m<v(t+2)$; but $\{e\}^{h}-(n)$ can be only one of $\{e\}^{u}(n)$ and $\{e\}^{w}(n)$, so that knowing $\{e\}^{h_{-}}$determines what $h_{-}$is on the interval.

This completes the proof of Lemma 2 .

THeOREM. Let $T$ be the upper semi-lattice of finite subsets of $N$. Then $T$ can be embedded as an initial segment in the upper semi-lattice of degrees of recursive unsolvability.

Proof. If $\left\{a_{0}<a_{1}<\cdots<a_{k-1}\right\}$ is a finite subset of $N$ then we shall have $\underline{h}_{a_{0} \cdots a_{k-1}}$ correspond to it, where $h$ is the function we are about to construct. Thus in particular $\underline{Q}$ will correspond to $\phi$. In order that this be an embedding of the finite subsets of $N$ as an initial segment of the degrees it is necessary and sufficient that:

(i) if $\left\{a_{0}, \cdots, a_{k_{-1}}\right\}$ and $\left\{b_{0}, \cdots, b_{r-1}\right\}$ are distinct sets, then $\underline{h}_{a_{0} \cdots a_{k-1}} \neq \underline{h}_{b_{0} \cdots b_{r-1}}$;

(ii) if $\left\{a_{0}, \cdots, a_{k-1}\right\}$ is included in $\left\{b_{0}, \cdots, b_{r-1}\right\}$, then $\underline{h}_{a_{0} \cdots a_{k-1}} \leqq$ $\underline{h}_{b_{0} \cdots b_{r-1}}$;

(iii) if $\underline{d}<\underline{h}_{a_{0} \cdots a_{k-1}}$, then for some $j, 0 \leqq j \leqq k-1$, we have $\underline{d} \leqq \underline{h}_{a_{0}} \cdots \hat{a}_{j} \cdots a_{k-1}$.

Note that (i) includes the statement that $h_{i}$ is not recursive, and together with (iii) implies that $h_{i}$ is of minimal degree. Note too that we need do nothing to guarantee that (ii) holds; it follows from the definition of $h_{a_{0} \cdots a_{k-1}}$.

We define a sequence $\left(f_{0}^{\alpha}, f_{0}^{\alpha}, g^{\alpha}\right)$ of special triples, each a con- 
traction of (or equal to) the preceding one, such that for each $\alpha, f_{0}^{\alpha}, f_{1}^{\alpha}$, and $g^{\alpha}$ are recursive.

Indeed define $f_{0}^{0}, f_{1}^{0}, g^{0}$ as follows:

$$
\begin{array}{rlrl}
g^{0}(0) & =0 & g^{0}(1)=1 \\
g^{0}(n+2) & =(\mu t)(E r)\left[t=p_{\phi(n)}^{r}+1 \Lambda g^{0}(n+1)<t\right] \\
f_{0}^{0}(m) & =0 & & \text { for all } m \\
f_{1}^{0}(m) & =1 & & \text { if } g^{0}(n+1) \leqq m<g(n+2) \Lambda(E r)\left(m=p_{\phi(n)}^{r}\right) \\
& =0 & & \text { otherwise. }
\end{array}
$$

Then clearly $\left(f_{0}^{0}, f_{1}^{0}, g^{0}\right)$ is a special triple and $f_{0}^{0}, f_{1}^{0}$, and $g^{0}$ are recursive functions.

We proceed inductively as follows:

Case 1. $\alpha+1$ is not of the form $22^{e} 3^{A} 5^{B} 7^{t}$, where $A$ and $B$ represent $\left\{a_{0}, \cdots, a_{k-1}\right\}$ and $\left\{b_{0}, \cdots, b_{r-1}\right\}$ in some standard coding, $a_{0}<\cdots<a_{k-1}, \quad b_{0}<\cdots<b_{r-1}$, and $t<2$. In this case we set $f_{0}^{\alpha+1}=f_{0}^{\alpha}, f_{1}^{\alpha+1}=f_{1}^{\alpha}$, and $g^{\alpha+1}=g^{\alpha}$.

Case 2. The hypothesis of Case 1 is false and $t=0$. In this case we want to guarantee that if $\left\{a_{0}, \cdots, a_{k-1}\right\}$ is not included in $\left\{b_{0}, \cdots, b_{r-1}\right\}$, then $h_{a_{0} \cdots a_{k-1}}$ is not recursive in $h_{b_{0} \cdots b_{r-1}}$ with Godel number $e$. Of course if $\left\{a_{0}, \cdots, a_{k-1}\right\}$ is included in $\left\{b_{0}, \cdots, b_{r-1}\right\}$, then we do not want to do this (good thing-we can't) so we proceed as in Case 1.

Let $j$ be minimal such that $a_{j} \notin\left\{b_{0}, \cdots, b_{r-1}\right\}$; we will use the fact that $\left(f_{0}^{\alpha}, f_{1}^{\alpha}, g^{\alpha}\right)$ is a special triple to find an interval in which the $a_{j}$ 'th function varies but for each $i$ the $b_{i}$ 'th function does not; we then compute a value for $\{e\}^{h}-\left(p_{a_{j}}^{x}\right)$ (where $\left.b_{0} \cdots b_{r-1}\right)$ and choose for $h\left(p_{a_{j}}^{x}\right)$ the opposite value. The end result is that for each $h \in F\left(f_{0}^{\alpha+1}, f_{1}^{\alpha+1}, g^{\alpha+1}\right), h_{a_{j}}$ is not recursive in $h_{b_{0} \cdots b_{r-1}}$ with Godel number $e$. Once this has been done for every $e$, we conclude that $h_{a_{0} \cdots a_{k-1}}$ is not recursive in $h_{b_{0} \cdots b_{r-1}}$, for $h_{a_{j}}$ is recursive in $h_{a_{0} \cdots a_{k-1}}$ but not in $h_{b_{0} \cdots b_{r-1}}$.

To do this we let

$$
\begin{aligned}
& n_{0}=(\mu n)(E m)\left[g^{\alpha}(m+1) \leqq n<g^{\alpha}(m+2) \Lambda(E t)\left(n=p_{\phi(m)}^{t}\right) \Lambda\right. \\
& \left.a_{j}=\phi(m) \Lambda f_{0}^{\alpha}(n) \neq f_{1}^{\alpha}(n)\right] ;
\end{aligned}
$$

$n_{0}$ exists since $\left(f_{0}^{\alpha}, f_{1}^{\alpha}, g^{\alpha}\right)$ is a special triple.

Subcase 1. $\sim(E w)\left[w \in F^{*}\left(f_{0}^{\alpha}, f_{1}^{\alpha}, g^{\alpha}\right) \wedge\{e\}^{w-}\left(n_{0}\right)\right.$ is defined]. In this case let $s$ be defined by $s(m)=f_{0}^{\alpha}(m)$ for $0 \leqq m<g^{\alpha}(2)$ and use Lemma 1 to get a special triple $\left(f_{0}^{\alpha+1}, f_{1}^{\alpha+1}, g^{\alpha+1}\right)$ satisfying the induction 
requirements. Note particularly, here and in the subsequent cases, that since these functions are recursive in $f_{0}^{\alpha}, f_{1}^{\alpha}, g^{\alpha}$ which are assumed to be recursive, we have that $f_{0}^{\alpha+1}, f_{1}^{\alpha+1}, g^{\alpha+1}$ are recursive.

Subcase 2. $(E w)\left[w \in F^{*}\left(f_{0}^{\alpha}, f_{1}^{\alpha}, g^{\alpha}\right) \wedge\{e\}^{w-}-\left(n_{0}\right)\right.$ is defined]. Let $v$ be the least such $w$ and let

$$
\begin{aligned}
& t_{0}=(\mu t)\left[t \in F^{*}\left(f_{0}^{\alpha}, f_{1}^{\alpha}, g^{\alpha}\right) \Lambda \ln t>n_{0} \Lambda \operatorname{lht} \geqq \operatorname{lh} v \Lambda\right. \\
& \quad(t)_{n_{0}} \neq\left\{e^{v}-\left(n_{0}\right) \Lambda(x)_{0 \leqq x<l h v}\left(P_{b_{0}}(x) \mathbf{V} \cdots \bigvee P_{b_{r-1}}(x) . \rightarrow(t)_{x}=(v)_{x}\right)\right] .
\end{aligned}
$$

Now apply Lemma 1 with $t_{0}$ as the initial segment to obtain $\left(f_{0}^{\alpha+1}, f_{1}^{\alpha+1}, g^{\alpha+1}\right)$.

Case 3. The hypothesis of Case 1 is false and $t=1$. In this case we wish to guarantee that if $d$ is recursive in $h_{a_{0} \cdots a_{k-1}}$ with Godel number $e$, then either $h_{a_{0} \cdots a_{k-1}}$ is recursive in $d$ or $d$ is recursive in some $h_{a_{0} \cdots \hat{a}_{j} \cdots a_{k-1}}$. But to do this we need only apply Lemma 2 with $e$ and $a_{0}, \cdots, a_{k-1}$, to get $\left(f_{0}^{\alpha+1}, f_{1}^{\alpha+1}, g^{\alpha+1}\right)$. (Note the role that the recursiveness of $f_{0}^{\alpha}, f_{1}^{\alpha}$, and $g^{\alpha}$ play here.)

This is the end of the construction. It is clear that there is exactly one function $h$ in the intersection of the sequence of special triples and that this function has exactly the properties we want. The enterprising soul will see that in fact we have embedded $T$ in the set of degrees $\leqq \underline{Q}^{\prime \prime}$.

Corollary. If $B$ is finite, then $P(B)$ and any initial segment of $P(B)$ is embeddable as an initial segment in the upper semilattice of degrees of unsolvability.

(This result has been obtained independently by J. Shoenfield.)

I expect that any finite partially ordered set with a least element can be embedded as an initial segment of the degrees and that this can be shown using methods not much more complicated than these. Indeed, at first glance, or even second glance, it might seem that slight modifications of the above construction give a proof of the conjecture. This is not so; indeed, technical difficulties appear out of nowhere, and they even make it impossible to construct a degree which is greater than exactly three distinct minimal degrees, and nothing else except $\underline{o}$. It remains to be seen whether these technical difficulties are really essential difficulties, or whether some simple trick will enable them to disappear. 


\section{REFERENCES}

1. Gerald E. Sacks, Degrees of Unsolvability, Princeton University Press, Princeton, New Jersey, 1963.

2. Clifford Spector, On degrees of recursive unsolvability, Ann. of Math. 64 (1956), 591-92.

Received April 19, 1966. This paper is part of the author's Doctoral dissertation, Cornell University, February 1966.

CoRNell UNIVERSITY

ITHACA, NEW YoRK 


\section{PACIFIC JOURNAL OF MATHEMATICS}

\section{EDITORS}

\section{H. ROYDEN}

Stanford University

Stanford, California

\section{J. P. Jans}

University of Washington

Seattle, Washington 98105

\section{J. DUGUNDJI}

Department of Mathematics

Rice University

Houston, Texas 77001

RICHARD ARENS

University of California

Los Angeles, California 90024

\section{ASSOCIATE EDITORS}
E. F. BeCKenbaCH
B. H. NeumanN
F. WOLF
K. YosIDA

\section{SUPPORTING INSTITUTIONS}

UNIVERSITY OF BRITISH COLUMBIA CALIFORNIA INSTITUTE OF TECHNOLOGY UNIVERSITY OF CALIFORNIA MONTANA STATE UNIVERSITY UNIVERSITY OF NEVADA NEW MEXICO STATE UNIVERSITY OREGON STATE UNIVERSITY UNIVERSITY OF OREGON OSAKA UNIVERSITY UNIVERSITY OF SOUTHERN CALIFORNIA
STANFORD UNIVERSITY UNIVERSITY OF TOKYO UNIVERSITY OF UTAH WASHINGTON STATE UNIVERSITY UNIVERSITY OF WASHINGTON

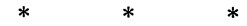

AMERICAN MATHEMATICAL SOCIETY CHEVRON RESEARCH CORPORATION TRW SYSTEMS

NAVAL ORDNANCE TEST STATION 


\section{Pacific Journal of Mathematics}

\section{Vol. 24, No. $1 \quad$ May, 1968}

Harry P. Allen, Lie algebras of type $D_{4}$ over algebraic number fields ...... 1

Charles Ballantine, Products of positive definite matrices. II............ 7

David W. Boyd, The spectral radius of averaging operators ............ 19

William Howard Caldwell, Hypercyclic rings ................... 29

Francis William Carroll, Some properties of sequences, with an application

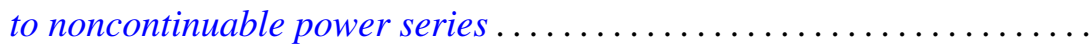

David Fleming Dawson, Matrix summability over certain classes of

sequences ordered with respect to rate of convergence ........... 51

D. W. Dubois, Second note on David Harrison's theory of preprimes. . . . . 57

Edgar Earle Enochs, A note on quasi-Frobenius rings.............. 69

Ronald J. Ensey, Isomorphism invariants for Abelian groups modulo bounded groups ................................ 71

Ronald Owen Fulp, Generalized semigroup kernels ................ 93

Bernard Robert Kripke and Richard Bruce Holmes, Interposition and approximation ................................. 103

Jack W. Macki and James Sai-Wing Wong, Oscillation of solutions to second-order nonlinear differential equations ..................

Lothrop Mittenthal, Operator valued analytic functions and generalizations



T. S. Motzkin and J. L. Walsh, A persistent local maximum of the pth power deviation on an interval, $p<1 \ldots \ldots \ldots \ldots \ldots \ldots \ldots \ldots \ldots \ldots . \ldots \ldots$

Jerome L. Paul, Sequences of homeomorphisms which converge to homeomorphisms ...........................

Maxwell Alexander Rosenlicht, Liouville's theorem on functions with elementary integrals.

Joseph Goeffrey Rosenstein, Initial segments of degrees .

$\mathrm{H}$. Subramanian, Ideal neighbourhoods in a ring ............

Dalton Tarwater, Galois cohomology of abelian groups . .

James Patrick Williams, Schwarz norms for operators ... .

Raymond Y. T. Wong, A wild Cantor set in the Hilbert cube. 